\title{
Nachruf auf Paul B. Baltes
}

Prof. Dr. Dr. h.c. mult. Paul B. Baltes, ein herausragender Psychologe unserer Zeit, ist am 7. 11. 2006 im Alter von 67 Jahren gestorben. Die Psychologie hat einen brillanten Wissenschaftler und eine wichtige Stimme verloren. Die Gerontologie beklagt den Verlust eines ihrer kraftvollsten und nachdrücklichsten Fürsprecher. Der Tod von Paul Baltes hinterlässt eine große Lücke, aber auch ein großes und starkes Netzwerk von WissenschaftlerInnen, die mit ihm zusammengearbeitet haben.

Paul Baltes wurde 1939 in Saarlouis geboren. Von 1959 bis 1963 studierte er an der Universität des Saarlandes Psychologie. Nach dem Diplom verbrachte er ein Jahr an der University of Nebraska. Dort lernte er K. Warner Schaie kennen. Nachdem er seine bahnbrechende methodologische Dissertation zum Problem der Entwirrung von Alters-, Kohorten- und Periodeneffekten auf die Entwicklung abgeschlossen hatte, nahm er das Angebot von K. Warner Schaie einer Professur an der University of West
Virginia an. Aus den wegweisenden „West Virginia Conferences on Lifespan Development“ entwickelte sich die Buchserie „Lifespan Development and Behavior“. In dieser Reihe unterzog eine stetig wachsende Gruppe von WissenschaftlerInnen die dominierende Auffassung der Entwicklungspsychologie als einer primär an Wachstumsprozessen und Kindheit orientierten Wissenschaft einer grundlegenden Kritik. Nach dieser Kritik umfasst Entwicklung die gesamte Lebensspanne von der Empfängnis bis ins hohe Alter (Baltes \& Goulet, 1970) und schließt Verluste ebenso ein wie Gewinne, Reifung ebenso wie Alterung, und biologische Einflusssysteme ebenso wie kulturelle. Paul Baltes war einer der Pioniere dieser theoretischen Orientierung in der modernen Entwicklungspsychologie (Baltes, Reese \& Lipsitt, 1980).

Im Alter von 33 Jahren ging Paul Baltes als Leiter an das College of Human Development der Pennsylvania State University. Die Max-Planck-Gesellschaft holte ihn dann 
1980 als Direktor ans Max-Planck-Institut für Bildungsforschung in Berlin. Als er 2004 den Status eines Emeritus erreichte, war dies für ihn, ganz im Sinne seiner Lehre, ein Übergang zu neuen Herausforderungen. Er wurde Distinguished Professor an der University of Virginia und Direktor des International Max Planck Research Network on Aging (MaxNet Aging), einem virtuellen Max-Planck-Institut (dem ersten seiner Art), das sich mit der Untersuchung sozial- und verhaltenswissenschaftlicher Aspekte des Alterns beschäftigt. Darüber hinaus blieb er bis zu seinem Lebensende Sprecher der International Max Planck Research School „The Life Course: Evolutionary and Ontogenetic Dynamics“ (LIFE) sowie Vorstandsmitglied der von der Deutschen Akademie der Naturforscher Leopoldina und dem Konvent für Technikwissenschaften der Union der deutschen Akademien der Wissenschaften (acatech) gegründeten Arbeitsgruppe „Chancen und Probleme einer alternden Gesellschaft - Die Welt der Arbeit und des lebenslangen Lernens“.

Paul Baltes hinterlässt ein Euvre von über 300 Veröffentlichungen, darunter 27 Bücher sowie die 26-bändige International Encyclopedia of the Social and Behavioral Sciences, die er zusammen mit Neil Smelser herausgab. Seine wissenschaftliche Anerkennung zeigt sich auch in vier Ehrendoktorwürden sowie zahlreichen Mitgliedschaften in angesehenen in- und ausländischen wissenschaftlichen Akademien. Für seine wissenschaftlichen Leistungen erhielt er 12 hochrangige Preise und Ehrungen von nationalen und internationalen Fachgesellschaften. So verlieh ihm die DGPs 2004 ihre höchste Auszeichnung, indem sie Paul Baltes für sein Lebenswerk ehrte.

Höchste Anerkennung erfuhr Paul Baltes auch über die wissenschaftliche Community hinaus. Als einziger Psychologe gehörte er dem Orden pour le mérite für Wissenschaften und Künste an, und er erhielt das Große Bundesverdienstkreuz mit Stern. Zudem wurde er in eine Gruppe berufen, die den Bundespräsidenten Horst Köhler zu Themen demografischen Alterns berät.

Wo soll man beginnen und wo enden, wenn man versucht, das Bild eines außerordentlichen Wissenschaftlerlebens nachzuzeichnen? Über wissenschaftliche Produktivität sowie professionelle und öffentliche Ehrungen hinaus zeichnen Paul Baltes fünf Glanzlichter aus: Internationalität, Interdisziplinarität, Generativität, gesellschaftliches Interesse, institutionelle Besonnenheit und Klugheit.

Internationalität: Bis in seine letzten Lebenstage baute Paul Baltes Brücken zwischen den Ländern und gründete internationale Netzwerke. Er war davon überzeugt, dass internationaler Austausch die Qualität wissenschaftlicher Arbeit erhöht und wissenschaftliche Erkenntnisse fördert. Nur wenige deutsche Wissenschaftler haben in den USA eine derart hohe Anerkennung gefunden.

Interdisziplinarität: Paul Baltes hatte sich als Spiritus Rector der Lebensspannen-Psychologie der interdisziplinären Untersuchung der menschlichen Entwicklung verschrieben. Dies zeichnete sich bereits während seiner Zeit als Department Head an der Penn State University ab. Schon damals leitete er eine interdisziplinäre Fakultät von Psychologen und Soziologen. Auch seine Arbeit im Social Science Research Council bot reichhaltigen Anlass zu interdisziplinärem Austausch. Er initiierte die Berliner Altersstudie (BASE), die biomedizinische, psychiatrische und psychologische mit soziologischen und ökonomischen Sichtweisen des Alterns verbindet. BASE wurde auch deshalb zum Erfolg, weil Paul Baltes es verstand, die verschiedenen disziplinären Ausrichtungen in einen gleichberechtigten Dialog zu führen. In seinen späteren Jahren freute es ihn, im Kontext der Akademien, denen er angehörte, und insbesondere im Orden pour le Mérite, Gespräche zu führen, die den fachwissenschaftlichen Horizont überschritten. Das Streben nach dem Renaissance-Ideal des allseits gebildeten Gelehrten verband er mit einem wachen Auge für die Erfordernisse und Entwicklungschancen moderner Gesellschaften.

Das dritte Glanzlicht: Die Arbeit von Paul Baltes war überdurchschnittlich früh und intensiv durch Generativität geprägt. Nach Erik Erikson ist die Fürsorge für nachfolgende Generationen, die Weitergabe von Einsichten und Erfahrungen die entscheidende Entwicklungsaufgabe der mittleren und späten Jahre. Paul Baltes nahm diese Herausforderung schon viel früher an. Von den Anfangsjahren seiner Karriere an war es ihm eine wichtige Aufgabe, Doktoranden und Postdoktoranden mit großem persönlichen Einsatz in vielen Lebensbereichen zu beraten und zu betreuen. Wir, die Autoren dieses Beitrags, hatten das große Glück, dass wir seine Studenten waren und von ihm als Mentor profitieren konnten. Bereits in den 1980er Jahren hat Paul Baltes mit anderen sowie mit Unterstützung der VW Stiftung ein Graduiertenkolleg in Deutschland gegründet. Eines seiner Lieblingsprojekte der späten Jahre war die Gründung einer Akademie der Wissenschaften für jüngere Wissenschaftler. Das Projekt wurde von der Berlin-Brandenburgischen Akademie der Wissenschaften und der Leopoldina, deren Vize-Präsident Paul Baltes war, gemeinsam umgesetzt. Und es passt zu dieser Facette seiner Persönlichkeit, dass Paul Baltes sich bis zuletzt im Kontext der International Max Planck Research School LIFE für die Unterstützung des wissenschaftlichen Nachwuchses eingesetzt hat.

Das vierte Glanzlicht: Paul Baltes, der Psychologe, der Gerontologe, der Wissenschaftler, zeigte Interesse an gesellschaftlichen Themen, und er ging an die Öffentlichkeit. Er war sich sicher, dass Psychologie etwas zu sagen hat und dass sie zur Lösung der Herausforderungen, denen moderne Gesellschaften ausgesetzt sind, beitragen kann. An die Öffentlichkeit zu gehen, ist jedoch nicht leicht - es ist eine Kunst, die viel Einsatz erfordert. Paul Baltes war nicht nur in dieser Hinsicht ein Künstler. Die gesellschaftliche Diskussion über lebenslanges Lernen, über die Folgen der demografischen Alterung, über die Chancen und Härten des höheren und höchsten Alters und über die Frage nach einem selbstbestimmten Sterben hat Paul Baltes vorangebracht und geprägt.

Das letzte Glanzlicht: Wissenschaft findet in Institutionen statt und wird durch institutionelle Eigenschaften 
und ihre Logik beeinflusst. Paul Baltes dachte und handelte über sein unmittelbares persönliches Interesse hinaus zum Nutzen der Institution und trug auf diesem Wege beispielsweise wesentlich zum Gedeihen und Ansehen des Max-Planck-Instituts für Bildungsforschung in Berlin bei. Über die Jahre übernahm er immer wieder Verantwortung, vor allem mit dem Ziel, die Spielräume der Verhaltens- und Sozialwissenschaften in der Max-Planck-Gesellschaft zu erweitern. Seine Motivation, Institutionen aufzubauen und zu formen, reichte auch in den Bereich privater Stiftungen. Paul Baltes spielte eine wichtige Rolle bei der höchsten Spende, die je in Europa einer Universität gestiftet wurde, nämlich jene 200 Millionen Euro, die die International University Bremen jüngst von der JacobsStiftung bekam.

Diese fünf Glanzlichter, die Paul Baltes als Person charakterisieren, sind durch die Höhepunkte seiner wissenschaftlichen Arbeit zu ergänzen. Niemand, der sich für die Kohorteneffekte bei der Untersuchung der menschlichen Entwicklung interessiert, kommt an Paul Baltes’ Arbeiten zu diesem Thema vorbei (z.B. Baltes, 1968). Paul Baltes hat die Programmatik der Psychologie der Lebensspanne geordnet und zum konzeptuellen Gerüst einer Entwicklungspsychologie im umfassenden Sinne erweitert. Schon früh interessierte er sich für das Potenzial kognitiver Funktionsfähigkeit bei Erwachsenen und im höheren Alter. Seine Arbeiten im Bereich der kognitiven Trainingsforschung haben sowohl den Fortbestand von kognitivem Entwicklungspotenzial im Alter als auch dessen Grenzen empirisch überzeugend nachgewiesen. Das Interesse an dem Potenzial des höheren Alters hat ihn auch veranlasst, sich an die psychologische Erforschung der Weisheit zu wagen. Hier hat er ein neues Erhebungsparadigma etabliert und unter anderem gezeigt, dass es nicht ausreicht, alt zu werden, um weise zu werden. Neben dem Potenzial war Paul Baltes auch an den Ursachen von Funktionsverlusten interessiert. Er hat nachgewiesen, dass Hören, Sehen und Gehen mit zunehmendem Alter vermehrte kognitive Ressourcen benötigen. Schließlich trat er in jüngerer Zeit immer mehr dafür ein, die Erforschung der Entwicklung des Verhaltens mit der Erforschung neuronaler Mechanismen und gesellschaftlicher Bedingungen zu verknüpfen. In diesem Zusammenhang sprach er auch von biokulturellen Ko-Konstruktivismus und warnte davor, das Verhalten zur abhängigen Variable des Gehirns zu erklären.

Gemeinsam mit seiner früh verstorbenen Frau Margret M. Baltes war er daran interessiert, Prozesse zu bestimmen, anhand derer es möglich ist, menschliche Entwicklung zu erklären und zu optimieren. Das von den beiden entwickelte SOK-Modell der Entwicklungsregulation, für das sich inzwischen die unterstützenden Befunde mehren, postuliert, dass Selektion, Kompensation und Optimierung die drei zentralen Regulationsmechanismen menschlichen Entwicklung sind (Baltes \& Baltes, 1990).

Paul Baltes hat ein reiches Erbe und allen, die ihn kannten, eine innere Stimme hinterlassen. Er wird die entwicklungspsychologische und gerontologische Forschung bis weit in die Zukunft hinein inspirieren. Er verstand es meisterhaft, Verbindungen herzustellen - im Wissenschaftlichen, im Wissenschaftspolitischen, im Gesellschaftlichen und im Persönlichen. Er hat die Vielschichtigkeit des menschlichen Lebenslaufs theoretisch, methodisch und forschungspraktisch durchdrungen wie kein zweiter. Deswegen beschränkten sich sein Wirken, sein Wissen und seine weltweite Anerkennung nicht auf seine Heimatdisziplin, die Entwicklungspsychologie, sondern zogen weite Kreise in die Geistes- und Naturwissenschaften. Paul Baltes hat die Entwicklungspsychologie als ebenbürtigen Partner im Kreis der Wissenschaften etabliert. Er stellte Fragen ins Offene und machte sie zum Ausgangspunkt einer neuen Forschungsidee, einer neuen Institution oder einer neuen Freundschaft. Seine Vorliebe galt dem Unfertigen und Verbesserbaren. Vielleicht besaß er auch deswegen den Mut, sich dem hohen Alter zuzuwenden, einer besonders unfertigen und verbesserbaren Phase des Lebens, die zu erreichen ihm selbst nicht vergönnt war.

Ursula M. Staudinger und Ulman Lindenberger 